\title{
Heterogeneous nucleation in a glass-forming alloy
}

\author{
J. J. Wall, ${ }^{1, \text { a) }}$ C. T. Liu, ${ }^{2}$ W.-K. Rhim, ${ }^{3}$ J. J. Z. Li, ${ }^{3}$ P. K. Liaw, ${ }^{2}$ H. Choo, ${ }^{2}$ and W. L. Johnson ${ }^{3}$ \\ ${ }^{1}$ LANSCE-LC, Los Alamos National Laboratory, Los Alamos, New Mexico 87545, USA \\ ${ }^{2}$ Department of Materials Science and Engineering, University of Tennessee, Knoxville, \\ Tennessee 37996, USA \\ ${ }^{3}$ Division of Engineering and Applied Science, California Institute of Technology, Pasadena, \\ California 91125, USA
}

(Received 6 May 2008; accepted 30 May 2008; published online 20 June 2008)

\begin{abstract}
Nucleation in the undercooled liquid state in the bulk metallic glass-forming composition $\mathrm{Zr}_{52.5} \mathrm{Cu}_{17.9} \mathrm{Ni}_{14.6} \mathrm{Al}_{10} \mathrm{Ti}_{5}$ (VIT-105), produced using high purity (PA) and commercial purity (CA) raw materials was investigated using electrostatic levitation and ex situ neutron diffraction. The CA material was observed to have a lower density than the PA sample and crystallized at relatively shallow undercooling. The densities of the samples at temperatures above the solidus showed an oxygen-dependent hysteresis associated with the state change, indicating the presence of oxygen-stabilized intermetallics. The PA alloy exhibited three distinct crystallization modes dependent on the thermal history of the melt, one of which showed a net volume expansion. (C) 2008 American Institute of Physics. [DOI: 10.1063/1.2948861]
\end{abstract}

It is well known that Zr-based bulk metallic glasses (BMGs) are inherently susceptible to oxygen contamination. In a general sense, the presence of oxygen is undesirable in Zr-based BMGs as it results in a reduced glass-forming ability, leading to crystallization during cooling from the liquid state. A number of recent studies have shown that a metastable face centered cubic (fcc) phase forms in a variety of Zr-based BMG-forming alloys upon reheating in the presence of oxygen. ${ }^{1-4} \mathrm{~A}$ more comprehensive understanding of the nucleation mechanisms that affect GFA and phase precipitation in BMG-forming alloys is necessary to further the current state of the understanding of glass formation.

Electrostatic levitation (ESL) provides a good environment in which to observe nucleation, as it decouples the sample from extrinsic heterogeneous nucleation sites. Previous ESL studies have identified the so-called overheating threshold in Zr-based BMGs, which is a superheating temperature, $T_{\mathrm{thr}}$, which must be exceeded prior to cooling or the materials will crystallize at shallow undercooling. ${ }^{5,6}$ This threshold temperature is systematically dependent on the oxygen concentration of the alloy. In this study, we present data to further characterize $T_{\text {thr }}$ and the mechanisms of nucleation in the bulk metallic glass-forming alloy VIT-105 $\left(\mathrm{Zr}_{52.5} \mathrm{Cu}_{17.9} \mathrm{Ni}_{14.6} \mathrm{Al}_{10} \mathrm{Ti}_{5}\right)$.

The samples used for this study were extracted from two separate heats, one produced using high purity elemental charge materials (Zr purity $>99.9$ at. \%) and the other produced using commercial grade materials (Zr purity $>99.0$ at. \%). The high purity alloy (PA) had an oxygen concentration of $560 \mathrm{ppm}$ (atomic herein), and the lower purity alloy (CA) had an oxygen concentration of $3000 \mathrm{ppm}$, as determined by wet chemical analyses. Note that these heats are the same materials as those used by Liu et al. in a previous study. ${ }^{7} 50 \mathrm{mg}$ extractions from the CA and PA heats were arc melted on a water-cooled $\mathrm{Cu}$ plate, forming spheroids, which were used for the ESL study.

\footnotetext{
${ }^{\text {a) }}$ Author to whom correspondence should be addressed. Tel.: +1-704-5952162. FAX: +1-704-595-2860. Electronic mail: jwall@epri.com. Present address: Electric Power Research Institute, Charlotte, NC 28262.
}

ESL was used to suspend the CA and PA spheroids, which were heated to the superheated liquid state using a Nd-doped yttrium aluminum garnet laser. A comprehensive description of the levitator can be found in Ref. 8. The molten levitated samples were free cooled by radiation into the supercooled-liquid state, at which point they crystallized. The sample temperatures were measured in real time using a two-color pyrometer. The specific volume evolution during cooling, crystallization, and remelting was measured from charge coupled device images of the sample periphery taken in situ. The threshold temperatures $\left(T_{\mathrm{thr}}\right)$ of each alloy were experimentally determined by heating to various temperatures and free cooling, noting the amount of undercooling before recalescense. One of the PA samples was heated to $T=T_{\text {thr }}$ and immediately free cooled. This sample and another PA sample (free cooled from above $T_{\text {thr }}$ ) were removed from the levitator and characterized using neutron scattering. The neutron scattering measurements were performed using the general purpose powder diffractometer at the Intense Pulsed Neutron Source (IPNS) facility at Argonne National Laboratory. The IPNS is a spallation neutron source, meaning that the entire diffraction pattern may be collected simultaneously by stationary detectors using polychromatic radiation. Additional information on neutron diffraction phase analysis may be found in available literature., ${ }^{9,10}$

Figure 1 shows a plot of the data points corresponding to overheating threshold temperatures, $T_{\mathrm{thr}}$, and their respective nucleation temperatures, $T_{\text {cry }}$, for the PA and CA VIT-105 alloys containing varying amounts of oxygen. The values obtained by Lin et al. ${ }^{6}$ for VIT-105 containing varying amounts of oxygen are included in the figure. The subscripts 1 and 2 for $T_{\text {cry }}$ indicate free cooling from above and below $T_{\text {thr }}$, respectively. Note how both $T_{\text {thr }}$ and $T_{\text {cryl }}$ are systematically dependent on the oxygen content, while $T_{\text {cry2 }}$ is invariant. It should be noted here that the values of $T_{\text {thr }}$ obtained in the current study are higher than those of the previous study. The reason for this discrepancy is unclear at present.

A comparison of the $V_{\mathrm{sp}}(T)$ behavior of the PA and CA alloys revealed that both materials exhibit varying degrees of volume hysteresis above the eutectic temperature [Figs. 2(a) 


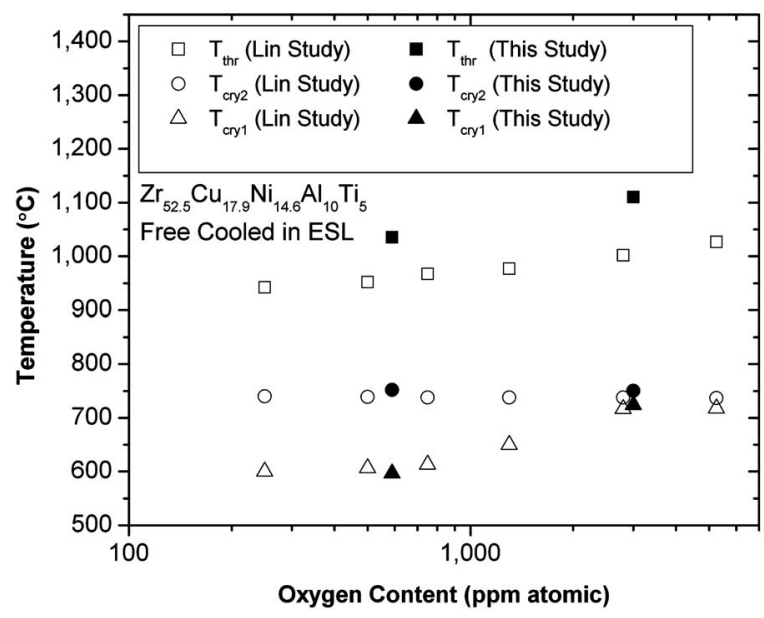

FIG. 1. The initial temperature threshold for maximum undercooling, $T_{\text {thr }}$, crystallization temperature for samples free cooled from below the threshold temperature, $T_{\text {cry2 }}$, and crystallization temperature for samples free cooled from above the threshold temperature, $T_{\text {cryl }}$ vs respective oxygen content in VIT-105 samples. The data from the current study are plotted with those from the study conducted by Lin et al. (Ref. 6).

and 2(b)]. This indicates that an intermetallic phase remains in the melt at temperatures above what would constitute the liquidus in an ideally pure sample $\left(T_{l}^{*}\right)$. The persistent intermetallic phase is then oxygen stabilized, which is evident from the relative magnitudes of hysteresis observed in the
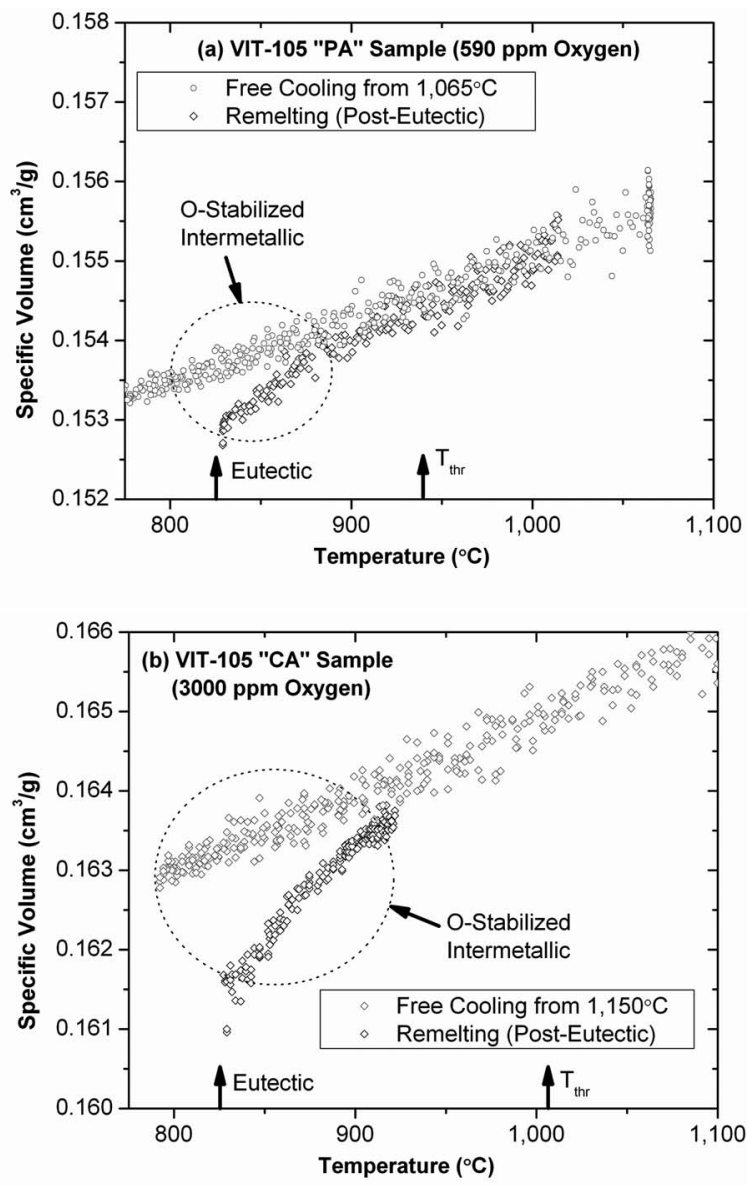

FIG. 2. (a) Specific volume of the VIT-105 PA sample cooled from well above $T_{\text {thr. }}$. (b) That presented in (a) for the CA alloy. Note the effect of oxygen on the magnitude of hysteresis of the density in the superheated liquid state.

Downloaded 20 Jun 2008 to 131.215.225.9. Redistribution subject
CA alloy (590 ppm O) and the PA alloy (3000 ppm O). As such, this oxygen-stabilized intermetallic phase is believed to be directly responsible for $T_{\mathrm{thr}}$. Under this scenario, at $T_{l}^{*}$, a majority of the eutectic phase has melted away, but rather than going into solution, the oxygen in the sample interstitially stabilizes a eutectic intermetallic phase (type 1 here). Further heating requires the oxygen to concentrate in the type-1 phase, thereby reducing its net volume fraction with increasing temperature. At some amount of superheating (corresponding to $T_{\mathrm{thr}}$ ), the oxygen concentration within the type-1 particles becomes too high for the structure to exist stably, and they transform into a different oxygen-stabilized intermetallic phase (type-2), which is structurally dissimilar to the eutectic phase. Note that if the oxygen-stabilized (eutecticlike) type- 1 phase were to melt at $T_{\text {thr }}$, it would reform upon cooling below $T_{\text {thr. }}$. To investigate the above mechanism in the high purity VIT-105 alloy, we shall further examine the PA alloy.

Three distinct crystallization modes were observed in the PA alloy during free cooling, dependent on the applied thermal history in the superheated liquid state: (1) an isothermal hold above $T_{\mathrm{thr}}$, (2) an isothermal hold below $T_{\mathrm{thr}}$, and (3) rapid heating to $T_{\mathrm{thr}}$ and immediately free cooling. Plots of $V_{\mathrm{sp}}(T)$ showing the free cooling, crystallization, and reheating are presented for cases 1-3 in Figs. 3(a)-3(c). Cases 1 and 2 showed exothermic crystallization at deep and shallow undercoolings, respectively, which is consistent with Fig. 1. In these cases, crystallization corresponds to a net decrease in the specific volume as the samples decrease their configurational entropy. In case 3, where the sample was heated to $T_{\text {thr }}$ and immediately free cooled, the sample dilated during solidification. This dilation suggests that the transformation at $T_{\text {thr }}$ involves an intermediate phase, having a low density relative to the type- 1 and type- 2 intermetallics, and which catalyzes the formation of a nonequilibrium, low-density intermetallic phase during undercooling.

The neutron diffraction measurements showed that the phases formed during recalescense in the PA alloy samples cooled from above $T_{\text {thr }}$ (case 1) and heated rapidly to $T_{\text {thr }}$, then immediately free cooled (case 3 ) were different. The case $3\left(+\Delta V_{\mathrm{sp}}\right)$ sample diffraction pattern was fitted using the Rietveld method ${ }^{11}$ with coexisting tetragonal phases. Least squares refinement of the fit revealed that the structures of these phases were $P 4_{2} / \mathrm{mmm}$ with $a=7.41 \AA$ and $c=6.66 \AA$, and $I 4 / \mathrm{mmm}$ with $a=3.20 \AA$ and $c=11.10 \AA$. The fit diffraction pattern is shown in Fig. 4. The case 1 sample diffraction pattern showed broad diffraction peaks of low intensity relative to the case 3 sample. Efforts to fit the Bragg peaks for the case 1 sample diffraction pattern using the Rietveld method were unsuccessful due to the weak character of the diffraction (Fig. 4). It can be stated with confidence, however, that the phases that formed in each of the PA samples were different. The primary crystalline component in the case 1 sample is likely tetragonal $\mathrm{Zr}_{2} \mathrm{Ni}$, which is a eutectic phase.

After remelting, at temperatures below $T_{\text {thr }}$, oxygen acts to stabilize the tetragonal $\mathrm{Zr}_{2} \mathrm{Ni}$ eutectic phase in the superheated liquid VIT-105. As such, both the PA and CA alloys contained an oxygen-stabilized eutectic nucleation catalyst at $T_{l}^{*}<T<T_{\text {thr. }}$. This claim is supported by the oxygen concentration independence of $T_{\text {cry2 }} . T_{\text {thr }}$, then, corresponds to the transformation of the oxygen-stabilized tetragonal $\mathrm{Zr}_{2} \mathrm{Ni}$. At $T_{\text {thr }}$, in a high purity alloy, an unstable primitive tetragonal 

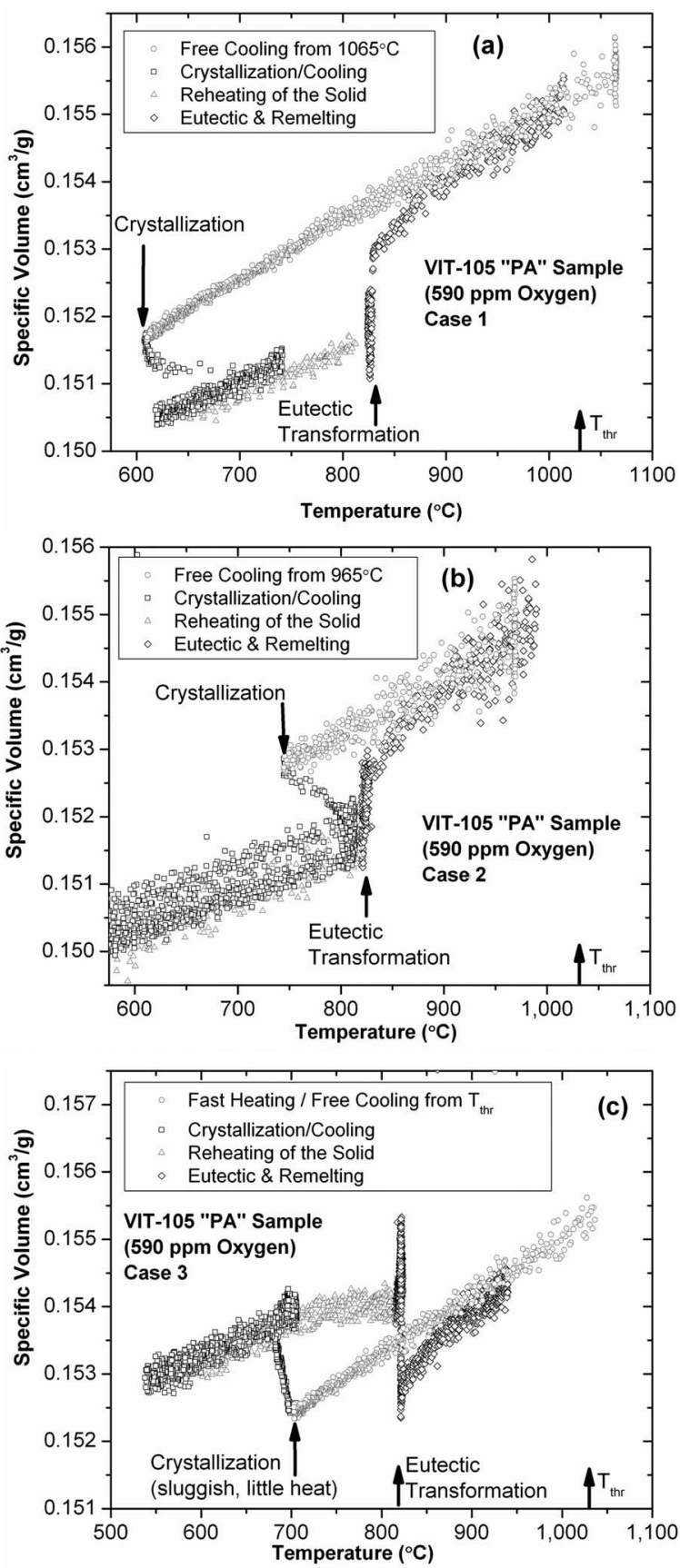

FIG. 3. (a) is the $V_{\mathrm{sp}}(T)$ of the PA alloy during a solidification-remelting cycle with an initial sample temperature of $T>T_{\text {thr }}$ in the ESL. (b) is the $V_{\text {sp }}(T)$ during a cycle with an initial sample temperature of $T<T_{\text {thr }}$ (c) is the $V_{\mathrm{sp}}(T)$ for the sample heated rapidly to $T_{\mathrm{thr}}$ and immediately free cooled, showing volume expansion.

phase forms, then decomposes into the type- 2 oxygenstabilized intermetallic. It seems plausible that the type-2 oxygen-stabilized intermetallic phase is a structural analog to fcc $\mathrm{Zr}_{2} \mathrm{Ni}$, which is known to be stabilized by oxygen at high temperatures, ${ }^{12,13}$ and has been reported to form in devitrified $\mathrm{Zr}$-based BMGs containing oxygen. The observed increase in $V_{\mathrm{sp}}$ in the case-3 PA alloy during solidification was unexpected. Shen et al. ${ }^{14}$ reported a volume increase during reheating of the $\mathrm{Pd}_{40} \mathrm{Cu}_{40} \mathrm{Ni}_{20}$ glass-forming alloy, and it was stated at the time that it was the only known glass-forming alloy to expand upon crystallization. The formation of the
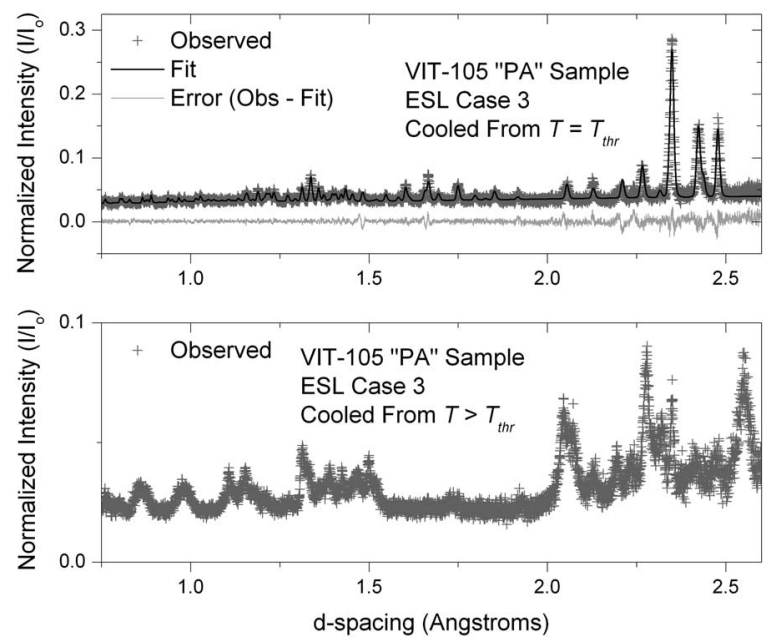

FIG. 4. The top part shows the case 3 sample neutron diffraction pattern and the rietveld fit. Note that the intensity errors in the fit were probably due to the small sample and relatively large crystallites. The bottom part shows the case 1 sample neutron diffraction pattern.

primitive tetragonal phase may then account for the irreversibility of transformation at $T_{\mathrm{thr}}$. The formation of the unstable primitive tetragonal phase at $T_{\mathrm{thr}}$ indicates that the oxygenstabilized type-1 phase occupies a saddle point in the Gibbsfree energy and, thus, is inherently metastable in the superheated liquid. As such, $T_{\text {thr }}$ corresponds to an activation energy for the transformation of the oxygen-stabilized eutectic phase into the structurally dissimilar type- 2 oxygenstabilized phase. As oxygen lowers the density of the liquid, above some nominal oxygen concentration, there are two possible scenarios: (1) the primitive oxygen-stabilized tetragonal phase becomes stable above $T_{\text {thr }}$ and acts as a catalyst for heterogeneous nucleation of the low-density tetragonal intermetallc at relatively shallow undercooling, or (2) the particle size of the type- 2 phase becomes large enough to favor heterogeneous nucleation of a noneutectic phase. It is expected that such a mechanism is critical to glass formation in many, if not all, Zr-based glass-forming alloys.

${ }^{1}$ A. Gebert, J. Eckert, and L. Schultz, Acta Mater. 56, 5475 (1998).

${ }^{2}$ J. Saida and A. Inoue, J. Non-Cryst. Solids 317, 97 (2003).

${ }^{3}$ M. F. de Oliveira, W. J. Botta, M. J. Kaufman, and C. S. Kiminami, J. Non-Cryst. Solids 304, 51 (2002).

${ }^{4}$ U. Koster, D. Zander, and R. Janlewing, Mater. Sci. Forum 386, 89 (2001).

${ }^{5}$ S. Mukherjee, Z. Zhou, J. Schroers, W. L. Johnson, and W. K. Rhim, Appl. Phys. Lett. 84, 5010 (2004).

${ }^{6}$ X. H. Lin, W. L. Johnson, and W.-K. Rhim, Mater. Trans., JIM 38, 473 (1997).

${ }^{7}$ C. T. Liu, M. F. Chisholm, and M. K. Miller, Intermetallics 10, 1105 (2002).

${ }^{8}$ W. K. Rhim, S. K. Chung, D. Barber, K. F. Man, G. Gutt, A. J. Rulison, and R. A. Spjut, Rev. Sci. Instrum. 64, 2961 (1993).

${ }^{9}$ S. J. L. Billinge, JOM 58, 47 (2006).

${ }^{10}$ A. D. Krawitz, Introduction to Diffraction in Materials Science and Engineering (Wiley, London, 2001).

${ }^{11}$ H. M. Rietveld, J. Appl. Crystallogr. 2, 65 (1969).

${ }^{12}$ R. Mackay, G. J. Miller, and H. F. Franzen, J. Alloys Compd. 204, 109 (1994).

${ }^{13}$ Z. Altounian, E. Batalla, J. O. Strom-Olsen, and J. L. Walter, J. Appl. Phys. 61, 149 (1987).

${ }^{14}$ T. D. Shen, U. Harms, and R. B. Schwarz, Appl. Phys. Lett. 83, 4512 (2003). 